\title{
A Performance Model for the HSDPA User Equipment and its Validation
}

\author{
Tien Van Do ${ }^{1}$ and Nam H. Do ${ }^{1}$ and Ram Chakka ${ }^{2}$ \\ ${ }^{1}$ Department of Telecommunications, \\ Budapest University of Technology and Economics \\ H-1117, Magyar tudósok körútja 2., Budapest, Hungary \\ Email: \{do,dohoai\}@hit.bme.hu \\ ${ }^{2}$ Meerut Institute of Engineering and Technology \\ Meerut, India \\ Email:ramchakka@yahoo.com
}

\begin{abstract}
A new queuing model is proposed for the performance evaluation of the High Speed Downlink Packet Access (HSDPA) protocol, with respect to a specified user, in UMTS networks. This analytical model is an integrated one capable of capturing many complicated features of HSDPA operation such as correlated and bursty traffic, channel fading, channel allocation policy and packet-losses in the air interface. The validation of the model for comparing the user terminal categories is performed with a detailed simulation of HSDPA terminals with "real" traffic traces. It is shown that the model is quite accurate to predict and compare the throughput of the HSDPA terminal categories.
\end{abstract}

Key words: HSDPA, Performance evaluation, Analytical model

\section{INTRODUCTION}

High Speed Downlink Packet Access (HSDPA) was introduced by the 3rd Generation Partnership Project (3GPP) to satisfy the demands for high speed data transfer in the downlink direction in UMTS networks. It can offer peak data rates of up to $10 \mathrm{Mbps}$, which is achieved essentially by the use of Adaptive Modulation and Coding (AMC), extensive multicode operation and a retransmission strategy [1].

However, efficient operation of HSDPA does require fast performance evaluation models in order to design, dimension, operate, maintain and update the system, both cost-effectively and efficiently. Such a performance model should be able to accommodate simultaneously all the important features and aspects pertaining to the operation of HSDPA, e.g., burstiness and the correlation amongst data traffic, channel assignments between voice and data traffic, channel coding schemes, as well as effects of the wireless environment such as channel fading.

In the literature, most works have used discrete event simulation to evaluate the performance of HSDPA [2][3][4]. Liu et al. [5] and Yang et al. [7] were the first to consider the interaction between queuing at the data link layer and AMC at the physical layer, in an analytical model. However, their analysis assumed Poisson arrivals at the data link layer and did not explicitly account for HSDPA. Moreover, we are aware of no work to date that attempts to quantitatively compare HSDPA user equipment (UE) categories. The problem is highly challenging, since we have to take into account a number of factors and characteristics such as

(i) the bursty and correlated nature of the packettraffic through the channels,

(ii) channel conditioning which is often represented by Channel Quality Indicator (CQI),

(iii) dynamic allocation of channels by a preset physical channel assignment scheme, and

(iv) packet-losses in the air interface due to fading channels. 


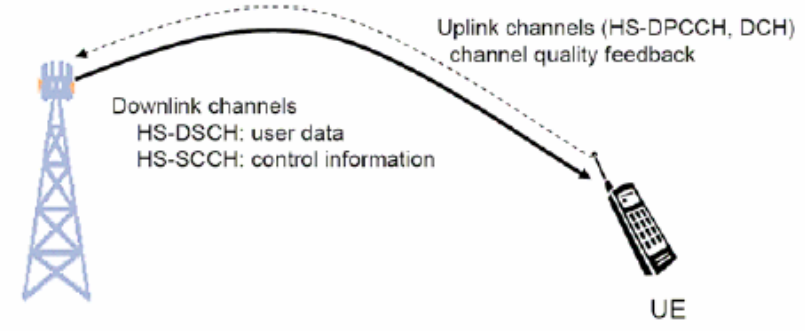

Node-B

Fig. 1: Channels in HSDPA

For this purpose, we develop a queuing model with the varying number of servers. This model is highly suitable to the problem that is tackled since (i) traffic correlations and burstiness can be represented by Markov modulation and by the use of Compound Poisson Processes Fig. 1: Channels in HSDPA (CPP), (ii) channel conditioning due to fading and the resulting CQI can be represented by a finite-state first-order Markov chain Z, (iii) dynamic channel allocation policy is represented by varying $c$ in the queuing model, modulated by an independent Markov process $\mathrm{U}$, (iv) packet losses in the air interface due to channel fading are modeled by negative arrivals. In [9], the first analytical model for HSDPA terminal without validation was presented. This paper proposes a refined performance model for HSDPA in the data link layer and also provides the validation of our model with a detailed simulation with real traffic traces and fading behaviour. In this respect, we show that the Compound Poisson Processes (CPP) and the simple parameter estimation of CPP from captured real traffic (Bellcore and Auckland traffic) can serve as an input parameter for the performance estimation of HSDPA. Recently there is a notable work by [8], where the authors propose the analytical approach to evaluate the throughput of HSDPA. However, they do not consider the stochastic nature of packet arrivals from user equipments and the specific parameters of user terminal categories. Therefore, the comparison of UE categories is difficult with their model. It is worth emphasizing that their model integrates some essential features of HSDPA such as the explicit equation for signal interference ratio and the Hybrid Automatic Repeat
Request. The integration of their model and our model is in progress, which will be reported in the companion report of this paper.

The rest of the paper is organised as follows. Section II provides an overview of HSDPA and Section III describes our proposed model for it. Numerical results are presented and discussed in Section IV and the paper concludes in Section V.

\section{HSDPA OPERATION}

In the implementation of HSDPA, several channels are introduced (Fig. 2). The transport channel carrying the user data, in HSDPA operation, is called the HighSpeed Downlink Shared Channel (HS-DSCH). The High-Speed Shared Control Channel (HS-SCCH), used as the downlink (DL) signaling channel, carries key physical layer control information to support the demodulation of the data on the HS-DSCH.

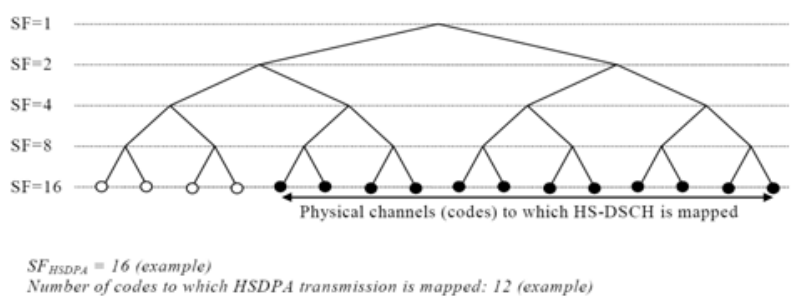

Fig. 2: HSDPA mapping to physical channels (3GPP TR 25.848)

The uplink (UL) signaling channel, called the HighSpeed Dedicated Physical Control Channel (HSDPCCH), conveys the necessary control data in the UL to Node B (Node B is responsible for the transmission and reception of data across the radio interface). User Equipment sends feedback information about the received signal ${ }^{1}$ quality on HS-DPCCH. That is, the UE calculates the DL Channel Quality Indicator (CQI) based on the received signal quality measured at the UE. Then, it sends the CQI on the HS-DPCCH channel to indicate which estimated transport block size,

1 In wireless communications, the quality of a received signal depends on a number of factors: the distance between the target and interfering base stations, the path-loss exponent, shadowing, channel-fading and noise. 
modulation type and number of parallel codes (i.e. physical channels) could be received correctly with reasonable block error rate in the DL. The CQI is integer valued, with a range between 0 and 30. The higher the CQI is, the better the condition of the channel and the more information can be transmitted.

Table 1: Modulation and max throughput when 5,10,15 codes are allocated for a specific user

\begin{tabular}{ccccc} 
Modulation & $\begin{array}{c}\text { Effective } \\
\text { code rate }\end{array}$ & \multicolumn{3}{c}{ Max. throughput Mbps } \\
QPSK & $1 / 4$ & 0.6 & 1.2 & 1.8 \\
QPSK & $2 / 4$ & 1.2 & 2.4 & 3.6 \\
QPSK & $3 / 4$ & 1.8 & 3.6 & 5.4 \\
16 QAM & $2 / 4$ & 2.4 & 4.8 & 7.2 \\
16 QAM & $3 / 4$ & 3.6 & 7.2 & 10.7
\end{tabular}

\section{A MODEL}

We consider a wireless connection between a specified wireless user and its Node- $B$, and assume that an ideal feedback channel exists.

\section{A. Assumptions}

1) Packet arrival process: In the Markovian framework, the autocorrelation of inter-arrival times are often modeled successfully by Markov-modulation. The packet arrival process at Node B is thus assumed to be modulated by a continuous time, irreducible Markov process, $X$, with $N_{X}$ states (phases) and $Q_{X}$ generator matrix. In [9 ][10][11], it has been successfully shown how Compound Poisson Processes (CPP [12]) and their superposition [9] can be used to model the burstiness in any given modulating phase. The arrival stream in each of the modulating phases of $X$ is thus assumed to follow the CPP. That is, the parameters of the GE interarrival time distribution of the packet arrival stream are $\left(\sigma_{i}, \theta_{i}\right)$ during the modulating phase $i$. Therefore, the probability distribution function of inter-arrival times $\tau_{i}$ during phase $i$ for the stream of packets is defined by $\operatorname{Pr}\left(\tau_{i}=0\right)=\theta_{i}$ and

$$
\operatorname{Pr}\left(0<\tau_{i}<t\right)=\left(1-\theta_{i}\right)\left(1-e^{-\sigma_{i} t}\right) .
$$

The rationale behind the choice GE distribution is follows. The parameters of the GE distribution are easily determined by the first two moments of sampled data. The GE distribution is the only distribution that is of least bias [12], if only the mean and variance are reliably computed from the samples.

2) CQI reporting process: In HSDPA, the UE calculates the Down Link (DL) Channel Quality Indicator (CQI) based on the received signal quality measured at the UE. Then, it sends the CQI (integer number) on the HS-DPCCH channel to indicate which estimated transport block size, modulation type and number of parallel codes (i.e.; physical channels) could be received correctly with reasonable block error rate in the DL. The higher the CQI is, the better the condition of the channel and the more information can be transmitted.

Since the CQI integer value sent by a UE varies between 0 and 30, a continuous time first-order Markov chain (called $Z$ ) of $N_{Z}=31$ states is used to model the CQI reporting process which depends on the fading channel dynamics.

3) Physical channel allocation for a single user: In HSDPA, a user may simultaneously utilise up to 15 codes (physical channels) in parallel. The available code resources are primarily shared in the time domain but it is possible to share the code resources using code multiplexing too. The number of available physical channels for a specified user is determined by the channel assignment scheme (i.e. the resource allocation policy), which takes into account traffic to and from other users. The dynamic allocation results in a varying number of available (allocated) channels, with respect to a given user. Since the arrival processes from other users are Markovian, the resource allocation is reasonably approximated by a Markovian process from the point of view of a specific user. We assume that the available channels for the specified user is modulated by a Markov process, called $U$, with $N_{U}$ states and $Q_{U}$ generator matrix. It is worth emphasizing that the calculation of $Q_{U}$ is not the focus of this paper. Furthermore, in the numerical study we will apply 
$N_{U}=1$ because we want to compare the performance of HSDPA user categories.

4) Packet-loss process in the air interface: It is shown in [13] that a Markovian approximation for the block error process can be a very good model for a broad range of parameters. Therefore, it is reasonable to model the packet loss in the air interface with the arrival stream of "negative packets". The arrival of one negative packet results in the loss of one packet in the air interface. In this paper, we assume the negative packets follow the GE distribution with parameters $\left(\rho_{i}, \delta_{i}\right)$. That is, the probability distribution function of inter-occurrence times $\left(\tau_{i}\right)$ of packet losses, strictly during phase $i$ is governed by $\operatorname{Pr}\left(\tau_{i}=0\right)=\delta_{i}$ and $\operatorname{Pr}\left(0<\tau_{i}<t\right)=\left(1-\delta_{i}\right)\left(1-e^{-\rho_{i} t}\right)$.

\section{B. A Performance Model}

As discussed above, the arrival process, the wireless channel-fading and the channel allocation are modulated by the independent Markov chains $X$ with $N_{X}$ states, $Z$ with $N_{Z}$ states and $U$ with $N_{U}$ states, respectively. If $Y$ denotes the effective Markov chain jointly modulating the arrival process, the wireless channel-fading and the number of channels allocated, then $Y$ can be determined quite easily from $X, Z$ and $U$, with $N_{Y}=N_{X} \times N_{Z} \times N_{U}$ states or phases. The generator matrix of $Y$ can be determined as the Kronecker sum of the generator matrices of $X, Z$ and $U$.

$$
Q_{Y}=Q_{X} \oplus Q_{Z} \oplus Q_{U} .
$$

Let the queuing capacity be $L$ at Node B which includes packets under service and packets waiting to be served. The state space of the queue formed at a Node- B at any time $t$ can be specified completely by two integer-valued random variables, $I(t)$ and $J(t)$. $I(t)$ varies from 1 to $N_{Y}$, representing the phase of the modulating Markov chain $Y$, and $0 \leq J(t)<L+1$ represents the number of positive customers in the system at time $t$, including any in service. The queue is now represented by a continuous time, discrete state Markov process, $\bar{V}$, on a rectangular lattice strip. Let $I(t)$, the phase, vary in the horizontal direction and $J(t)$, the queue length or level, in the vertical direction. We denote the steady state probabilities by $\left\{p_{i, j}\right\}$, where $p_{i, j}=\lim _{t \rightarrow \infty} \operatorname{Prob}(I(t)=i, J(t)=j)$, and let $\mathbf{v}_{j}=\left(p_{1, j}, \ldots, p_{N, j}\right)$.

To obtain the steady state probabilities $\left(\left\{p_{i, j}\right\}\right)$ and the performance measures, either the direct solution of the balance equations or the methodology presented in [10][11] can be used.

\section{NUMERICAL STUDY}

Twelve UE categories have been defined according to a number of factors that include the maximum number of HS-DSCH simultaneously received multicodes (5, 10 or 15), the minimum inter-TTI (Transmission Time Interval) time between the beginning of two consecutive transmissions to a specific UE, the maximum number of HS-DSCH transport block bits received within an HS-DSCH TTI, and the modulations (QPSK only or both QPSK and 16QAM) used (the interested user can check the 3GPP document [14] for more detail on the UE categories).

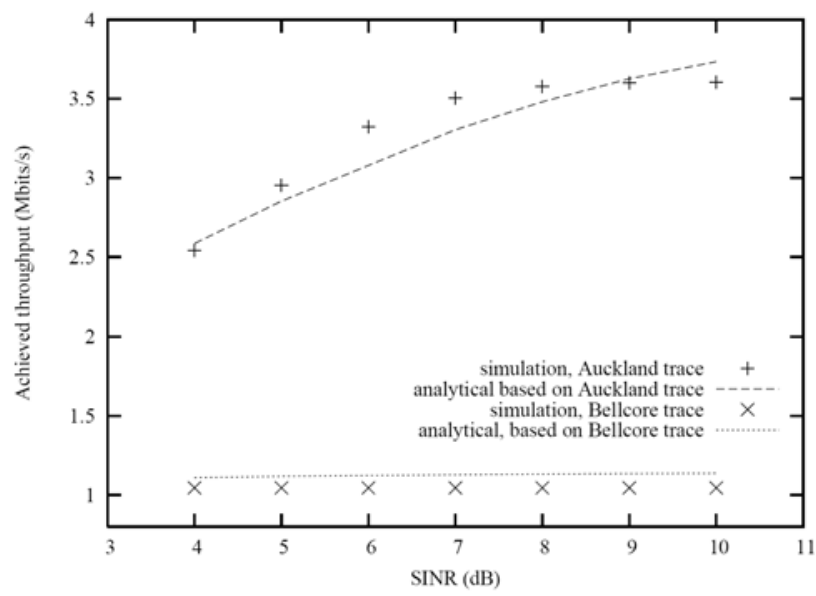

Fig. 4: UE category 10: Analytical and simulation results (throughput in Mbps). 


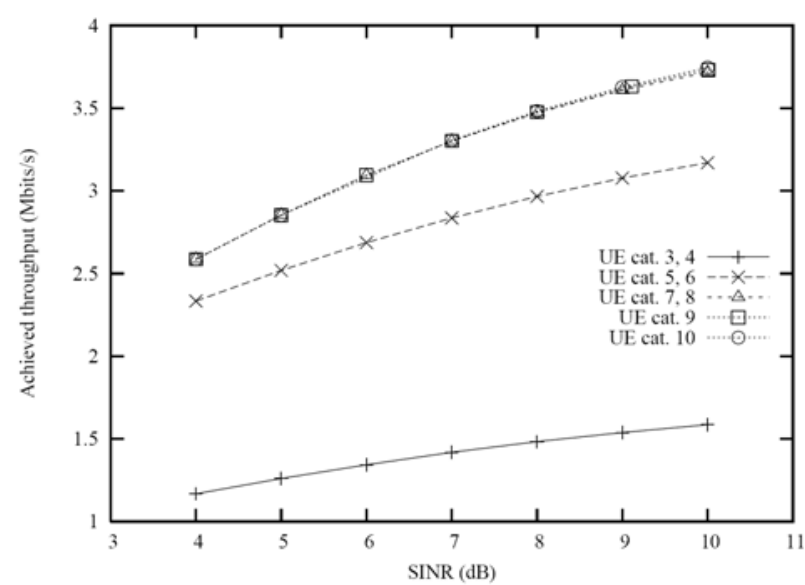

Fig.5: Achieved throughput vs. SINR with the Auckland traffic trace

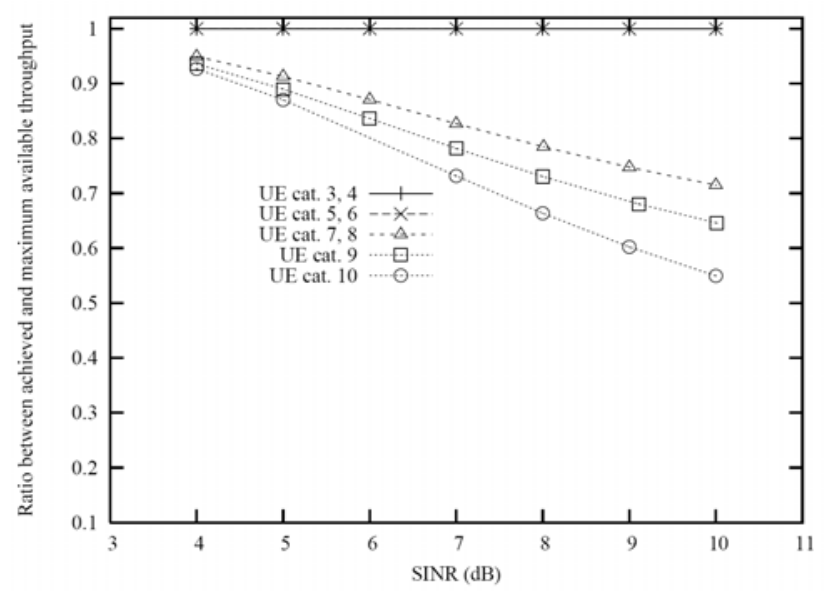

Fig. 6: Ratio between achieved and maximum throughput vs. SINR

In this paper, the UE categories 5, 6, 7, 8, 9 and 10 with inter-TTI $=1$ and UE categories $(3,4)$ with interTTI $=2$ are studied. Based on the CQI mapping table for UE categories specified in [14], UE categories 3 and 4, UE categories 5 and 6, UE categories 7 and 8 have the same characteristics. Therefore, 5 groups of UE categories are investigated.

The purpose of this study is to compare the UE categories, so we investigate a scenario where data is transferred from a network to a specified UE (with $N_{U}=1$ and $N_{X}=1$ ) and no packet loss is assumed in the evaluation. Note that the investigation of the channel allocation and the impact of packet loss in the air interface is the topic of future work.

The queuing capacity at the Node- $\mathrm{B}$ of this specified UE is assumed to be 150 packets. Traffic is assumed to follow the GE distribution with parameter pair $(\sigma, \theta)$, which are calculated based on the method of moment matching from the two traces of real traffic: the Bellcore traffic trace BC-pAug89 available from the Internet Traffic Archive [15] and the Auckland Internet traffic trace [16]. We also assume that the UE travels with a speed of $3 \mathrm{kmph}$. Therefore, the maximum Doppler frequency of the UE is $f_{d}=5.6 \mathrm{~Hz}$ at $2 \mathrm{GHz}$ carrier frequency. The service parameters (related to the variable packet sizes) are also determined from the traces similarly as done in [6].

To validate our model, we use the EURANE tool (http://www.ti-wmc.nl/eurane) which is an HSDPA simulator based on ns-2. It is worth emphasizing that the simulator is directly driven by the traffic traces (containing the interarrival of packets and the real size of packets) and the fading channel behaves according to the Nakagami- $m$ distribution function. To produce analytical results, the GE parameters of the input traffic and the GE service parameters are estimated from the monitored packet lengths in the trace and the transport block sizes of each UE at a specific CQI value [14], and the Markov process is used to approximate the Nakagami- $m$ distribution function. That is, we calculate the matrix $Q_{Z}$ of size $31 \times 31$ (note that $Q_{Z}$ depends on $m$ and the average SINR). Assume that the received signal-to-noise ratio (SINR), $\gamma$, of the fading channel has the Nakagami- $m$ (or Rice) probability density function [17 ]

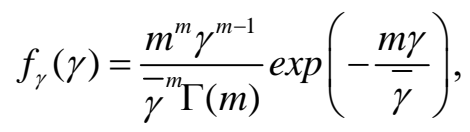

where $\bar{\gamma}=E(\gamma)$ is the average received SINR and $\Gamma(m)=\int_{0}^{\infty} t^{m-1} \exp (-t) d t$ is the Gamma Function with parameter $m$. Note that $m$ is the Nakagami fading 
parameter $(m \geq 1 / 2)$. Then a continuous time firstorder Markov chain (called $Z$ ) of $N_{Z}$ states can be used to approximate the fading channel dynamics. That is, the SINR in state $S_{i}$ is associated with $\gamma \in\left[\gamma_{i}, \gamma_{i+1}\right)$, the interval corresponds to a CQI value reported by a specific UE to its Node-B $\left(\gamma_{1}=0, \gamma_{N_{Z}+1}=\infty\right)$. Since the CQI integer value sent by a UE varies between 0 and $30, N_{Z}=31$. The CQI corresponding to the fading channel state $S_{i}$ is $C Q I=i-1$, for $i=1,2, \ldots, N_{Z}$. Based on the relation ([17]) between CQI and SINR

$$
C Q I= \begin{cases}0 & \text { SINR } \leq-16 \\ \left\lfloor\frac{\operatorname{SINR}}{1.02}+16.62\right\rfloor & -16<\mathrm{SINR}<14 \\ 30 & \text { SINR } \geq 14\end{cases}
$$

we determine $\mathrm{E}\left(\gamma_{i}\right)=\operatorname{SINR}\left(\forall i=1, \ldots, N_{Z}\right)$ for each $\left[\gamma_{i}, \gamma_{i+1}\right)$. Then $\gamma_{i}$ can be computed by solving the following equations

$$
E\left(\gamma_{i}\right)=\int_{\gamma_{i}}^{\gamma_{i+1}} \gamma f_{\gamma}(\gamma)
$$

The elements of the generator matrix, $Q_{Z}$, can be determined as follows

$$
\begin{aligned}
& Q_{Z}(k, k+1)=\aleph_{k+1} / \pi_{k} \quad(k=1,2, \ldots, 30) \\
& Q_{Z}(k, k-1)=\aleph_{k} / \pi_{k} \quad(k=2,3, \ldots, 31),
\end{aligned}
$$

where the level crossing rate $\left(\aleph_{n}\right)$ of mode $n$ (the AMC mode $n$ is chosen when the channel is in state $S_{n}$ ) is defined as in [18]:

$$
\begin{gathered}
\aleph_{n}=\sqrt{2 \pi \frac{m \gamma_{n}}{\bar{\gamma}}} \frac{f_{d}}{\Gamma(m)}\left(\frac{m \gamma_{n}}{\bar{\gamma}}\right)^{m-1} \exp \left(-\frac{m \gamma_{n}}{\bar{\gamma}}\right), \\
(n=1,2, \ldots, 31)
\end{gathered}
$$

and

$$
\pi_{k}=\int_{\gamma_{k}}^{\gamma_{k+1}} f_{\gamma}(\gamma) d \gamma
$$

$f_{d}$ is the mobility-induced Doppler spread.

In Fig. 4, we plot the curves of achieved throughput of UE category 10 vs SINR for $m=1$ and both traffic traces. It can be observed that our model can provide a good estimation (the relative error is around 5\%) for the throughput performance of UEs (the similar observation can be obtained with other UE categories).

In what follows, we present the results related to the Auckland traffic trace (the same observation can also be drawn with the Bellcore traffic trace). In Fig. 5, we plot the achieved throughput vs UE categories and SINR for the Auckland traffic trace. Based on the numerical study, we can state that UE category 7, 8, 9 and 10 have the same throughput performance.



Fig.7: PDF of CQI at the average of SINR $4 d B$ and $10 \mathrm{~dB}$

However, when we plot the efficiency ratio between the achieved average throughput and the maximum available average throughput (which latter is calculated assuming there are always packets to be transmitted at Node B) in Fig. 6, a different phenomenon is observed. UE of higher categories did not fully exploit the capability of the HSDPA channel. It is interesting that the higher the average SINR level is (Fig. 7), the lower the efficiency ratio is. From the viewpoint of the efficient usage of network scare resource (the interest of the network operators), it raises a need for the power control to be applied at the Node B. The power control should take into account the amount of traffic to be sent 
to the UEs. For the power control purpose, our model with the online estimation of the GE traffic parameters can be used to optimize the efficient usage of the radio resource.

\section{v. CONCLUSIONS}

We have proposed a framework to evaluate the performance of HSDPA. We present numerical results to compare the HSDPA categories, which is compared against the results obtained with more detailed simulation model of HSDPA based on the EURANE tool and real traffic traces. We also show the simple parameter estimation of CPP based on the moment matching from the traffic trace can give a good performance estimation for HSDPA. Further investigation includes the impact of the loss in the radio interface and the channel allocation scheme with the use of the analytical framework.

\section{REFERENCES}

[1] 3GPP Technical Report 25.848, version 4.0.0: Physical layer aspects of UTRA High Speed Downlink Packet Access. (March 2001).

[2] Brouwer, F., de Bruin, I., Silva, J.C., Souto, N., Cercas, F., Correia, A.: Usage of Link-Level Performance Indicators for HSDPA Network-Level Simulations in EUMTS. In: ISSSTA2004, Sydney, Australia. (augustusseptember 2004).

[3] Kolding, T., Frederiksen, F., Mogensen, P.: Performance Aspects of WCDMA Systems with High Speed Downlink Packet Access (HSDPA). In: VTC 2002, Vancouver. Volume 1. (September 2002) 477-481.

[4] Pedersen, K.I., Lootsma, T.F., Stottrup, M., Frederiksen, F., Kolding, T.E., Mogensen, P.E.: Network Performance of Mixed Traffic on High Speed Downlink Packet Access and Dedicated Channels in WCDMA . In: VTC 2004, Vancouver. Volume 6. (September 2004) 4496-4500.

[5] Liu, Q., Zhou, S., Giannakis, G.B.: Queuing With Adaptive Modulation and Coding Over Wireless Links: Cross-Layer Analysis and Design. IEEE Trans. on Wireless Communications 4(3) (May 2005) 1142-1153.

[6] H. T. Tran: MPLS Edge Nodes with Ability of Multiple LSPs Routing: Novel Adaptive Schemes and Performance Analysis. Research, Development and Application on Electronics, Telecommunications and
Information Technology, (Vietnamese Journal on Information Technologies and Communications, Series 3), pp. 39-53, 6/2008.

[7] Yang, L.L., Hanzo, L.: Improving the Throughput of DSCDMA Systems Using Adaptive Rate Transmissions Based on Variable Spreading Factors. In: Proceeding of VTC 2002, Vancouver. Volume 1. (September 2002) 1816-1820.

[8] Assaad, M. Zeghlache, D.: Analytical Model of HSDPA Throughput Under Nakagami Fading Channel. IEEE Transactions on Vehicular Technology, doi: 10.1109/TVT.2008.926609.

[9] Do, T.V., Chakka, R., Harrison, P.G.: An integrated analytical model for computation and comparison of the throughputsof the umts/hsdpa user equipment categories. In: MSWiM '07: Proceedings of the 10th ACM Symposium on Modeling, analysis, and simulation of wireless and mobile systems, New York, NY, USA, ACM (2007) 45-51

[10] Chakka, R., Do, T.V.: Some new Markovian models for traffic and performance analysis in telecommunication networks, Tutorial Paper. In Kouvatsos, D.D., ed.: Proceedings of the Second International Working Conference on Performance Modelling and Evaluation of Heterogeneous Networks (HET-NETs 04), Ilkley, UK (July 2004) T6/1-31.

[11] Chakka, R., Do, T.V.: MM $\quad \sum_{k=1}^{K} C P P_{k} / G E / c / L G \quad$-Queue with Heterogeneous Servers: Steady state solution and an application to performance evaluation. Performance Evaluation 64 (March 2007) 191-209.

[12] Kouvatsos, D.: Entropy maximisation and queueing network models. Annals of Operations Research 48 (1994) 63-126.

[13] Zorzi, M., Rao, R.R., Milstein, L.B.: Error Statistics in Data Transmission over Fading Channels. IEEE Trans. Commun. 46 (11) (November 1998) 1468-1477.

[14] 3GPP Technical Report 25.214, version 7.0.0: Physical layer procedures (FDD). (March 2006).

[15] The Internet Traffic Archive - http://ita.ee.lbl.gov

[16] Auckland Internet Traffic Capture:

[17] http://www.wand.net.nz/wand/wits/auck/6/20010612060000-e1

[18] Simon, M.K., Alouini, M.S.: Digital Communication over Fading Channels, Second Edition. John Wiley \& Sons, Inc. (2005).

[19] Wang, H.S., Moayeri, N.: Finite-State Markov channel-a useful model for radio communication channels . IEEE Transactions on Vehicular Technology (1995) 163-171. 


\section{AUTHORS' BIOGRAPHY}

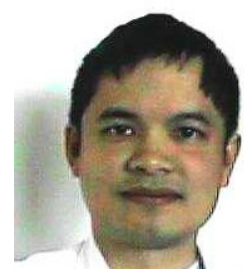

Tien Van Do received the M.Sc. and Ph.D. degrees in telecommunications engineering from the Technical University of Budapest, Hungary, in 1991 and 1996, respectively. He is an associate professor in the Department of Telecommunications of the
Middlesex University (UK), Norfolk State University (NSU, USA), Sri Sathya Sai Institute of Higher Learning (India) and RGMCET (India). At NSU, Dr. Chakka was awarded the Certificate of Excellence for Outstanding Scholarship from the School of Science and Technology. He published over 40 papers in Performability Modeling and Evaluation of Computing Systems, Communication Networks and Other Discrete Event Systems. Dr. Chakka is a member of IEEE and also IEEE Vehicular Technology Society.

Technical University of Budapest, and a leader of Communications Network Technology and Internetworking Group. He has participated in the COPERNICUS-ATMIN 1463, the FP4 ACTS AC310 ELISA, FP5 HELINET, FP6 CAPANINA projects funded by EC, and lead various projects on network planning, software implementations (ATM \& IP network planning software, GGSN tester, program for IMS performance testing, VoIP measurement,...), test and performance evaluation with NOKIA, T-COM, NOKIA and Siemens Networks, and industry partners. He was the person in charge for the RFI (Request for Information) and the technical specification of the public procurement worth of 2 MEuro for the testbed (IMS, UMTS, WiFi, etc,...) of Mobile Innovation Center in Budapest. His research interests are queuing theory, telecommunication networks, performance evaluation and planning of telecommunication networks.

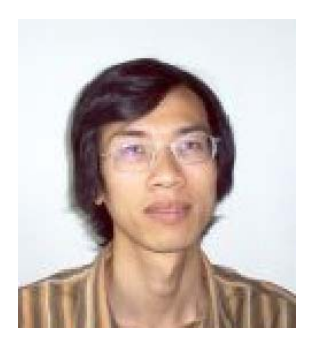

Do Hoai Nam received the M.Sc. in telecommunications engineering from the Technical University of Budapest, Hungary, in June 2006. He is currently a $\mathrm{PhD}$ student at the same university. His research interests include quality of service in wireless networks, the performance evaluation and planning of cross layered wireless systems, and scheduling algorithms for wireless networks.

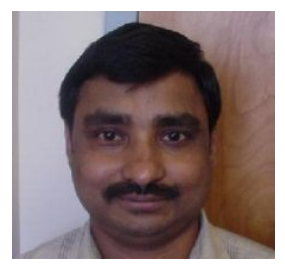

Ram Chakka received his B.Engg. (Electrical and Electronics, 1980), M.S. (Engg) (Computer Science and Automation, 1986), both from the Indian Institute of Science, Bangalore, India and Ph.D. (Computer Science, 1995) from the University of Newcastle upon Tyne, UK. Presently he is a Professor in Computer Science and Engineering and Director Research at MIET, Meerut, India. Earlier, he worked at Indian Institute of Science, University of Newcastle upon Tyne, Imperial College (London), 